\title{
Event classification from the Urdu language text on social media
}

\author{
Malik Daler Ali Awan ${ }^{\text {Corresp., }}{ }^{\text {, Nadeem Iqbal kajla }}{ }^{2}$, Amnah Firdous ${ }^{3}$, Mujtaba Husnain ${ }^{4}$, Malik Muhammad Saad \\ Missen ${ }^{4}$ \\ 1 Department of Software Engineering, Faculty of Computing, The Islamia University of Bahawalpur, Bahawalpur, Punjab, Pakistan \\ 2 Department of Information Technology, Muhammad Nawaz sharif university of agriculture, Multan, Multan, Punjab, Pakistan \\ 3 Computer Science and Information Technology, The Govt. Sadiq College and Women University Bahawalpur, Bahawalpur, Punjab, Pakistan \\ 4 Department of Information Technology, Faculty of Computing, The Islamia University of Bahawalpur, Bahawalpur, Punjab, Pakistan \\ Corresponding Author: Malik Daler Ali Awan \\ Email address: daler.ali@iub.edu.pk
}

The real-time availability of the internet has engaged millions of users around the world. The usage of regional languages is being preferred for effective and ease of communication that is causing multilingual data on social networks and news channels. People share ideas, opinions, and events that are happening globally i.e., sports, inflation, protest, explosion, and sexual assault, etc. in regional (local) languages. Extraction and classification of events from multilingual data have become bottlenecks because of resource lacking. In this research paper, we presented the event classification for the Urdu language text existing on social media and the news channels by using machine learning classifiers. The dataset contains more than 0.1 million $(102,962)$ labeled instances of twelve (12) different types of events. The title, its length, and last-4-words of a sentence are used as features to classify the events. The Term Frequency-Inverse Document Frequency ( $t f-i d f$ ) showed the best results as a feature vector to evaluate the performance of the six popular machine learning classifiers. The Random Forest (RF) and K-Nearest Neighbor (KNN) are among the classifiers that out-performed among other classifiers by achieving $98.00 \%$ and $99.00 \%$ accuracy, respectively. The novelty lies in the fact that the features aforementioned are not applied, up to the best of our knowledge, in the event extraction of the text written in the Urdu language. 
1 Event classification from the Urdu language text on 2 social media

3 Malik Daler Ali Awan ${ }^{1}$, Nadeem Iqbal ${ }^{2}$, Amnah Firdous ${ }^{3}$, Mujtaba Husnain ${ }^{1}$, and Malik

4 Muhammad Saad Missen ${ }^{1}$

5

$6 \quad{ }^{1}$ The Islamia University Bahawalpur of Bahawalpur, 61300, Pakistan

7 'Muhammad Nawaz sharif university of agriculture, Multan, 60000, Pakistan

$8{ }^{3}$ The Government Sadiq College Women University Bahawalpur, 61300, Pakistan

9

10

Corresponding Author:

Daler Ali, The Islamia University of Bahawalpur, Pakistan

Email address: daler.ali@iub.edu.pk

\section{Abstract}

The real-time availability of the internet has engaged millions of users around the world. The usage of regional languages is being preferred for effective and ease of communication that is causing multilingual data on social networks and news channels. People share ideas, opinions, and events that are happening globally i.e., sports, inflation, protest, explosion, and sexual assault, etc. in regional (local) languages. Extraction and classification of events from multilingual data have become bottlenecks because of resource lacking. In this research paper, we presented the event classification for the Urdu language text existing on social media and the news channels by using machine learning classifiers. The dataset contains more than 0.1 million $(102,962)$ labeled instances of twelve (12) different types of events. The title, its length, and last-4-words of a sentence are used as features to classify the events. The Term Frequency-Inverse Document Frequency $(t f-i d f)$ showed the best results as a feature vector to evaluate the performance of the six popular machine learning classifiers. The Random Forest (RF) and K-Nearest Neighbor (KNN) are among the classifiers that out-performed among other classifiers by achieving $98.00 \%$ and $99.00 \%$ accuracy, respectively. The novelty lies in the fact that the features aforementioned are not applied, up to the best of our knowledge, in the event extraction of the text written in the Urdu language.

\section{Introduction}

In the current digital and innovative era, text is still the strongest and dominant source of communication instead of pictures, emoji, sounds, and animations [1]. The innovative environment of communication; real-time availability [2] of the Internet and unrestricted access for communication on social networks have attracted billions of people around the world. Now, people are hooked together via the Internet like a global village because of the Internet. They preferred to share detailed worthy information about different topics, opinions, views, ideas, and events [3] on social networks in different languages. The usage of different languages is being popular because social media and news channels have created space for local languages [4]. 
40 Google input too ${ }^{1}$ provides language transliteration support for more than 88 different 41 languages. Many other tools like the software (Inpage and Pak-Urdu for the Urdu language) 42 provide the support to use local languages on social media for communication. The google 43 language translator ${ }^{2}$ is a platform that facilitates multilingual users of more than 100 languages

44 for conversation. Generally, people prefer to communicate in local languages instead of non45 local languages for sake of easiness.

46 A cursive language Urdu is one of the local languages that is being highly adapted for 47 communication. There are more than 300 million [10] Urdu language users all around the world 48 that can speak, understand and write in the Urdu language. The Urdu language is a mix-

49 composition of different languages i.e., Arabic, Persian, Turkish, and Hindi [11]. In Pakistan and 50 India, more than 65 million people can speak, understand, and write the Urdu language [12]. It is 51 one of the resource-poor, neglected languages [13] and the national language [14] of Pakistan:

52 the $6^{\text {th }}$ most populous ${ }^{2}$ country in the world. Urdu is also widely adopted and spoke as a second 53 language all over Pakistan [11-14].

54 In South Asia other countries [15] i.e., Bangladesh, Iran, and Afghanistan also have a 55 considerable number of Urdu language users. Pak Urdu Installer ${ }^{3}$ and Inpage are also common 56 software, it support the Urdu language for textual writing (communication).

57 In contrast to cursive languages, there exists noteworthy work of information extraction and 58 classification for i.e., English, French, German, and many other non-cursive languages [14-15].

59 Sifting worthy insights from an immense amount of heterogeneous text existing on social media 60 is an interesting and challenging task of Natural Language Processing (NLP). Event extraction 61 and classification is one of the NLP tasks. The information of event classification is helpful to 62 develop various NLP applications i.e., to respond to emergencies, outbreaks, rain, flood, and 63 earthquake [5], etc. Generally, people share their intent, appreciation, or criticism [6] i.e., 64 enjoying discount offers by selling brands or criticizing the quality of the product. Earlier 65 awareness of sentimental insights can be helpful to protect from business losses. The 66 implementation of smart- cities possess a lot of challenges; decision making, event management, 67 communication, and information retrieval. Extracting useful insights from an immense amount 68 of text, dramatically enhance the worth of smart cities [7]. Event information can be used to 69 predict the effects of the event on the community, improve security and rescue the people.

70 Furthermore, classification of events can be used to collect relevant information about a specific 71 topic, top-trends, stories, text summarization, and question and answering systems [8-9]. Such 72 information can be used to predict upcoming events, situations, and happening. For example, 73 protesting events reported on social media generally end with conflict among different parties, 74 injuries, death of people, and misuse of resources that cause anarchy. Some proactive

\footnotetext{
1 https://www.google.com/inputtools/

2 https://translate.google.com/?hl=en

2 https://www.worldometers.info/world-population/population-by-country/

${ }^{3}$ http://www.mbilalm.com/download/pak-urdu-installer.php
} 
75

76

77

78

79

80

81

82

83

84

85

86

87

88

89

90

91

92

93

94

95

96

97

98

99

100

101

102

103

104

105

106

107

108

109

110

111

112

113

114

measurements can be taken by the state to diffuse the situation and to prevent conflict. Similarly, event classification is crucial to monitor the law-and-order situation of the world. Extracting and classification of event information from Urdu language text is a unique, interesting, and challenging task. The characteristic features of the Urdu langue that made the event classification tasks more complex and challenging are listed below.

- Cursive nature of the script

- Morphologically enriched

- Different structures of grammar

- Right to the left writing style

- No text capitalization

Similarly, the lack of resources i.e., the Part of speech tagger (PoS), words stemmer, datasets, and word annotators are some other factors that made the processing of the Urdu text complex. There exist a few noteworthy works related to the Urdu language text processing (See the literature for more details). All the above-mentioned factors motivated us to explore Urdu language text for our task.

\section{Concept of Events}

The definition of events varies from domain to domain. In literature, the event is defined in various aspects, such as a verb, adjective, and noun based depending on the environmental situation [16-17]. In our research work event can be defined as "An environmental change that occurs because of some reasons or actions for a specific period and influences the community." For example, the explosion of the gas container, a collision between vehicles, terrorist attacks, and rainfall, etc. There are several hurdles to process Urdu language text for event classification. Some of them are i.e., determining the boundary of events in a sentence, identifying event triggers, and assigning an appropriate label.

\section{Event Classification}

"The automated way of assigning predefined labels of events to new instances by using pretrained classification models is called event classification.”. Classification is supervised machine learning; all the classifiers are trained on label instances of the dataset.

\section{Multiclass Event Classification}

It is the task of automatically assigning the most relevant one class from the given multiple classes. Some serious challenges of multiclassification are sentences overlapping in multiple classes [18-19] and imbalanced instances of classes. These factors generally affect the overall performance of the classification system.

\section{Lack of Recourse}

The researchers of cursive languages in the past were unexcited and vapid [13] because of lacking resources i.e., dataset, part of speech tagger and word annotators, etc. Therefore, a very low amount of research work exists for cursive language i.e., Arabic, Persian Hindi, and Urdu [20]. But now, from the last few years, cursive languages have attracted researchers. The main reason behind the attraction is that a large amount of cursive language data was being generated rapidly over the internet. Now, some processing tools also have been developed i.e., Part of 
115 speech tagger, word stemmer, and annotator that play an important role by making research 116 handier. But these tools are still limited, commercial, and close domain.

117 Natural language processing is tightly coupled with resources i.e., processing resources, datasets, 118 semantical, syntactical, and contextual information. Textual features i.e., Part of Speech (PoS) 119 and semantic are important for text processing. Central Language of Engineering (CLE) ${ }^{4}$ 120 provides limited access to PoS tagger because of the close domain and paid that diverged the 121 researcher to explore Urdu text more easily.

122 Contextual features [21] i.e., grammatical insight (tense), and sequence of words play important 123 role in text processing. Because of the morphological richness nature of Urdu, a word can be 124 used for a different purpose and convey different meanings depending on the context of contents. 125 Unfortunately, the Urdu language is still lacking such tools that are publicly available for 126 research. Dataset is the core element of research. Dataset for the Urdu language generally exists 127 for name entity extraction with a small number of instances that are

128

129

130

131

132

133

134

135

136

137

138

139

140

141

142

143

144

145

146

147

148

149

150

151

152

- Enabling Minority Language Engineering (EMILLE) (only 200000 tokens) [22].

- Becker-Riaz corpus (only 50000 tokens) [23]

- International Joint Conference on Natural Language Processing (IJCNLP) workshop corpus (only 58252 tokens)

- Computing Research Laboratory (CRL) annotated corpus (only 55,000 tokens are publicly available data corpora. [24]

There is no specific dataset for events classification for Urdu language text.

\section{Concept of Our System}

The overall working process of our proposed framework is given in Fig.1.

\section{Our Contribution}

- In this research article, we claim that we are the first ones who are exploring the Urdu language text to perform multi-class event classification at the sentence level using a machine learning approach,

- A dataset that is larger than state-of-art used in experiments. In our best knowledge classification for twelve 12 different types of events never performed,

- A comprehensive and detailed comparison of six machine learning algorithms is presented to find a more accurate model for event classification for the Urdu language text.

\section{Our Limitations}

- There is no specific Word2Vec model for Urdu language text,

- There is also no availability of the free (open source) Part of Speech tagger and word stemmer for Urdu language text,

- Also, there exists no publicly available dataset of Urdu language text for sentence classification.

\section{Related Work}

${ }^{4}$ http://www.cle.org.pk/ 
153 Classification of events from the textual dataset is a very challenging and interesting task of 154 Natural Language Processing (NLP). An intent mining system was developed [6] to facilitate 155 citizens and cooperative authorities using a bag of token model. The researchers explored the 156 hybrid feature representation for binary classification and multi-label classification. It showed a $1576 \%$ to $7 \%$ improvement in the top-down feature set processing approach. Intelligence information 158 retrieval plays a vital role in the management of smart cities. Such information helps to enhance 159 security and emergency management capabilities in smart cities [7]. The textual content on social

160

161

162

163

164

165

166

167

168

169

170

171

172

173

174

175

176

177

178

179

180

181

182

183

184

185

186

187

188

189

190

191 media is explored in different ways to extract event information. Generally, the event has been defined as a verb, noun, and adjective [14]. Event detection is a generic term that is further divided into event extraction and event classification. A combined neural network of the convolutional and recurrent network was designed to extract events from English, Tamil, and Hindi languages. It showed $39.91 \%, 37.42 \%$ and $39.71 \% \mathrm{~F} \_$Measure [17].

In the past, the researchers were impassive in cursive language, therefore a very limited amount of research work exists in cursive language i.e., Arabic, Persian Hindi, and Urdu [25]. Similarly, in the work of [25], the authors developed a multiple minimal reduct extraction algorithm which is an improved version of the Quick reduct algorithm [26]. The purpose of developing the algorithm is to produce a set of rules that assist in the classification of Urdu sentences. For evaluation purposes, an Arabic-based corpus containing more than 2500 documents was plugged in for classifying them into one of the nine classes. In the experiment, we compared the results of the proposed approach when using multiple and single minimal reducts. The results showed that the proposed approach had achieved an accuracy of $94 \%$ when using multiple reducts, which outperformed the single reduct method which achieved an accuracy of $86 \%$. The results of the experiments also showed that the proposed approach outperforms both the K-NN and J48 algorithms regarding classification accuracy using the dataset on hand.

Urdu textual contents were explored [27] for classification using the majority voting algorithm.

They categorized Urdu text into seven classes i.e., Health, Business, Entertainment, Science, Culture, Sports, and Wired. They used 21769 news documents for classification and reported $94 \%$ precision and recall. Dataset evaluated using these algorithms, Linear SGD, Bernoulli Naïve Bayes, Linear SVM, Naïve Bayes, random forest classifier, and Multinomial Naïve Bayes. A framework [28] proposed a tweet classification system to rescue people looking for help in a disaster like a flood [29]. The developed system was based on the Markov Model achieve 81\% and $87 \%$ accuracy for classification and location detection, respectively. The features used in their system are [29]:

- Number of words in a tweet (w)

- Verb in a tweet by (verb)

- Number of verbs in a tweet by (v)

- Position of the query by (Pos)

- Word before query word (before)

- Word after query word (after) 
192 To classify Urdu news headlines [30] by using maximum indexes of vectors. They used stemmed

193

194

195

196

197

198

199

200

201

202

203

204

205

206

207

208

209

210

211

212

213

214

215

216

217

218

219

220

221

222

223

224

225

226

227

and non-stemmed textual data for experiments. The system was specifically designed for text classification instead of event classification. The proposed system achieved $78.0 \%$ for competitors and $86.6 \%$ accuracy for the proposed methodology. In comparison, we used sentences of Urdu language for classification and explored the textual features of sentences. We have explored all the textual and numeric features i.e., title, length, last-4-words, and the combinations of these (for more detail see Tab. 1) in detail in this paper that were not reported ever in state-of-art according to our knowledge.

Twitter [31] to detect natural disasters i.e., bush fires, earthquakes and cyclones, and humanitarian crises [32]. To be aware of emergencies situation in natural disasters a framework work designed based on SVM and Naïve Bayes classifiers using word unigram, bi-gram, length, number of \#Hash tag, and reply. These features were selected on a sentence basis. SVM and Nave Bayes showed $87.5 \%$ and $86.2 \%$ accuracy respectively for tweet classification i.e., seeking help, offering for help, and none. A very popular social website (Twitter) textual data was used [33] to extract and classify events for the Arabic language. Implementation and testing of Support Vector Machine (SVM) and Polynomial Network (PN) algorithms showed promising results for tweet classification $89.2 \%$ and $92.7 \%$. Stemmer with PN and SVM magnified the classification $93.9 \%$ and $91.7 \%$ respectively. Social events [34] were extracted assuming that to predict either parties or one of them aware of the event. The research aimed to find the relation between related events. Support Vector Machine (SVM) with kernel method was used on adopted annotated data of Automated Content Extraction (ACE). Structural information derived from the dependency tree and parsing tree is utilized to derive new structures that played important role in event identification and classification. The Tweet classification of the tweets related to the US Air Lines [40] is performed by the sentiment analysis companies that are not related to our work. We tried to classify events at sentence level that is challenging since the Urdu sentence contains very short features as compared to a tweet. It is pertinent to mention that the sentiment classification is different from the event classification. Multiclass event classification is reported [41] comprehensively, deep learning classifiers are used to classify events into different classes.

\section{Materials \& Methods}

Event classification for Urdu text is performed using a supervised machine learning approach. A complete overview of the multi-class event classification methodology is given in Fig.1. Textual data classification possesses a lot of challenges i.e., word similarity, poor grammatical structure, misuse of terms, and multilingual words. That is the reason, we decided to adopt a supervised classification approach to classify Urdu sentences into different categories.

\section{Data Collection}

Peer] Comput. Sci. reviewing PDF | (CS-2020:11:55370:4:0:NEW 13 Oct 2021) 
228 Urdu data were collected from popular social networks (Twitter), famous news channel blogs 229 i.e., Geo News ${ }^{5}$, Urdu Point ${ }^{6}$, and BBC Urdu ${ }^{7}$. The data collection consists of the title, the main 230 body, the published date, the location, and the URL of the post. In the phase of data collection, a 231 PHP-based web scraper is used to crawl data from the above-mentioned social websites. A 232 complete post is retrieved from the websites and stored in MariaDB (database). Our dataset 233 consists of 0.1 million $(102,960)$ label sentences of different types of events. All the different 234 types of events used in our research work and their maximum number of instances are shown 235 below in Fig. 2.

236 There are twelve different types of events that we try to classify in our research work. These 237 events are a factual representation of the state and the situation of the people. In Fig. 2. 238 imbalances number of instances of each event are given. It can be visualized that politics, sports,

239

240

241

242

243

244

245

246

247

248

249

250

251

252

253

254

255

256

257

258

259

260

261

262

263

264 and Fraud \& Corruption have a higher number of instances while Inflation, Sexual Assault, and Terrorist attacks have a lower number of instances. These imbalanced numbers of instances made our classification more interesting and challenging.

Multiclass events classification tasks are comprised of many classes. The different types of events that are used in our research work i.e., sports, Inflation, Murder \& Death, Terrorist attacks, Politics, Law and Order, Earthquake, Showbiz, Fraud \& Corruption, Weather, Sexual Assault, and Business. All the sentences of the dataset are labeled by the above-mentioned twelve (12) different types of events. Finally, a numeric (integer) value is assigned to each type of event label (See Tab. 2 for more details of the label and its relevant numeric value).

\section{Preprocessing}

The initial preprocessing steps are performed on the corpus to prepare it for machine learning algorithms. Because textual data cannot directly process by machine learning classifiers. It also contains many irrelevant words. The detail of all the preprocessing steps is given below. These steps were implemented in a PHP-based environment. While the words tokenization is performed using the scikit library [20] in python.

\section{Post Splitting}

The PHP crawler extracted the body of the post. It comprises many sentences as a paragraph. In the Urdu language script, sentences end with a sign called "- "Hyphen (Khatma-تمب). It is a standard punctuation mark in the Urdu language to represent the end of the sentence. As mentioned earlier, we are performing event classification at the sentence level. So, we split paragraphs of every post into sentences. Every line in the paragraphs ending at Hyphen is split as a single line.

\section{Stop Words Elimination}

Generally, those words that occur frequently in text corpus are considered as stop words. These words merely affect the performance of the classifier. Punctuation marks (“!”, “@”," \#”, etc.) and frequent words of the Urdu languages ( $\left(\checkmark(\mathrm{ka}), \_\right.$(kay), $s(\mathrm{ki})$, etc.) are the common

\footnotetext{
5 https://urdu.geo.tv/

${ }^{6}$ https://www.urdupoint.com/daily/

7 https://www.bbc.com/urdu
} 
265

266

267

268

269

270

271

272

273

274

275

276

277

278

279

280

281

282

283

284

285

286

287

288

289

290

291

292

293

294

295

296

297

298

299

300

301

302

303

304

examples of stop words. All the stop words [28] that do not play an influential role in event classification for the Urdu language text are eliminated from the corpus. Stop words elimination reduces memory and processing utilization and makes the processing efficient.

\section{Noise Removal and Sentences Filtering}

Our data were collected from different sources (see section 3). It contains a lot of noisy elements i.e., multilanguage words, links, mathematical characters, and special symbols, etc. To clean the corpus, we removed noise i.e., multilingual sentences, irrelevant links, and special characters.

The nature of our problem confined us to define the limit of words per sentence. Because of the multiple types of events, it is probably hard to find a sentence of the same length. We decided to keep the maximum number of sentences in our corpus. All those sentences which are brief and extensive are removed from our corpus. In our dataset lot of sentences varying in length from 5 words to 250 words. We decided to use sentences that consist of 5 words to 150 words to lemmatize our research problem and to reduce the consumption of processing resources.

\section{Sentence Labeling}

In supervised learning, providing output (Label) detail in the corpus is a core element. Sentence labeling is an exhausting task that requires deep knowledge and an expert's skill of language. All the sentences were manually labeled by observing the title of the post and body of sentences by Urdu language experts (see Tab. 2 for sentence labeling). Three Urdu language experts were engaged in the task of sentence labeling. One of them is Ph.D. (Scholar) while the other two are M.Phil. To our best knowledge, it is the first largest labeled dataset for the multi-class event in the Urdu language.

\section{Feature Selection}

The performance of prediction or classification models is cohesively related to the selection of appropriate features. In our dataset six (6) features excluding "Date" as a feature are considered valuable to classify Urdu news sentences into different classes. All the proposed features that are used in our research work are listed in Tab.1.

\section{Why were these features selected?}

\section{Last- 4-Words of Sentence}

Occurrence, happening, and situations are generic terms that are used to represent events. In general, "verb" represents an event. The grammatical structure of Urdu language is Subject_ Object_Verb (SOV) [31], which depicts that verb, is laying in the last part of the sentences. For example, the sentence (“"حمد نـ بودون كو بانى ديا. Ahmad ney podon ko pani dia"), (Ahmad watered the plants) follows the SOV format. "Pani dia- بـانى ديانى" is the verbal part of the sentence existing in the last two words of the sentence. It shows the happening or action of the event. Our research problem is to classify sentences into different classes of events. So, that last_4_words are considered one of the vital features to identify events and non-event sentences. For example, in Tab. 3 in the event column underline/highlighted part of the sentence represents the happening of an event i.e., last_4_words in the sentence. While labeling the sentences we are strictly concerned that only event sentences of different types should be labeled.

\section{Title of Post}


305 Every conversation has a central point i.e., title. Textual, pictorial, or multimedia content that is 306 posted on social networks as a blog post, at the paragraph level or sentence level describes the 307 specific event. Although many posts contain irrelevant titles to the body of the message.

308 However, using the title as a feature to classify sentences is crucial because the title is assigned 309 to the contents-based material.

310 Length of Sentence

311 A sentence is a composition of many words. The length of the sentence is determined by the total

312 number of words or tokens that exist in it. It can be used as a feature to classify sentences

313 because many sentences of the same event have probably the same length.

314 Title and Length

315 The proposed feature is the combination of the title of the post and the length of the sentence.

316 The title represents the central idea of the post, and the length of the sentence varies from title to 317 title.

318 Title and Last-4-words

319 The combination of title and last_4_words in Urdu language text is very helpful to classify the 320 sentences. Because last_4_words generally represent the occurrence/happening of some event.

321 Length and Last-4-words

322 We also consider the combination of length with last_4_words as a valuable feature because the 323 length of a sentence varies from event to event.

324 Features Engineering

325 Feature Engineering is a way of generating specific features from a given set of features and 326 converting selected features to machine-understandable format. Our dataset is text-based that consists of more than 1 million (102,960 labeled) instances i.e., sports, inflation, death, terrorist

328 attack, and sexual assault, etc. 12 classes.

329 As mentioned earlier that the Urdu language is one of the resource-poor languages and since 330 there are no pre-trained word embedding models to generate the embedding vectors for Urdu 331 language text, we could not use the facility of Word2Vec embedding technique.

332 All the textual features are converted to numeric format i.e., (Term Frequency_Inverse 333 Document Frequency) TF_IDF and Count-Vectorizer. These two features TF_IDF and Count334 Vectorizer are used in a parallel fashion. The scikit-learn package is used to transform text data 335 into numerical value [20].

336 Count_Vectorization

337 The process of converting words to numerical form is called vectorization. Its working strategy

338 is based on term frequency. It counts the frequency of specific word $w$ and builds the spare 339 matrix-vector using bag-of-words (BOW). The length of the feature vector depends on the size 340 of the bag-of-words i.e., dictionary.

341 Term Frequency Inverse Document Frequency

342 It is a statistical measure of word $w$ to understand the importance of that word for specific

343 document $d$ in the corpus. The importance of a word is proportionally related to frequency i.e., 
344 higher frequency more important. The mathematical formulas related to TF_IDF are given

345 below:

346 Term Frequency $(\mathrm{TF})=\frac{\text { Number of time term } t \text { appears in document }}{\text { Total number of terms in documents }}$

347 Inverse Document Frequency (IDF) $=\log _{e} \frac{\text { Total number of document }}{\text { Total number of documents term t appears }}$

$348 \quad$ TF $I D F=T F * I D F$

349 Experimental Setup

350 Classifiers are the algorithms used to classify data instances into predefined categories. Many 351 classifiers exist that process the textual data using a machine learning approach. In our research 352 work, we selected the six most popular machine learning algorithms i.e., Random Forest (RF) 353 [10], K-Nearest Neighbor (KNN), Support Vector Machine (SVM, Decision Tree (DT), Naïve 354 Bayes Multinomial (NBM), and Linear Regression (LR).

355 Machine Learning Classifiers

356 In this section, we presented the detail of six classifiers that were used to classify the Urdu

357 sentences using different proposed features.

3581 Random Forest (RF)

359 This model is comprised of several decision trees that act as a building block of RF. Every

360 decision tree is created using the rules i.e., if then else, and the conditional statements, etc. [10].

361 These rules are then followed by the multiple decision trees to analyze the problem at a discrete 362 level.

$3632 k$-Nearest Neighbor

364 It is one of the statistical models that find the similarity among the data points using Euclidean

365 distance [35]. It belongs to the category of lazy classifiers and is widely used for classification 366 and regression tasks.

$367 \quad 3 \quad$ Support Vector Machine

368 It is based on statistical theory [36], to draw a hyperplane among points of the dataset. It is

369 highly recommended for regression and classification i.e., binary classification, multiclass

370 classification, and multilabel classification. It finds the decision boundary to identify different

371 classes and maximize the margin.

3724 Decision Tree

373 It is one of the supervised classifiers that work following certain rules. Data points/inputs are

374 split according to the specific condition [37]. It is used for regression and classification using the

375 non-parametric method because it can handle textual and numerical data. Learning from data

376 points is accomplished by approximating the sine curve with the combination of an if-else-like

377 set of rules. The accuracy of a model is related to the deepness and complexity of rules.

$3785 \quad$ Naïve Bayes Multinominal

379 It is a computationally efficient classifier for text classification using discrete features. It can also

380 handle the textual data by converting it into numerical [38] format using count vectorizer and

381 term frequency-inverse document frequency (tf- idf).

$3826 \quad$ Linear Regression 
383 It is a highly recommended classifier for numerical output. It is used to perform prediction by 384 learning linear relationships between independent variables (inputs) and dependent variables 385 (output) [39].

386 Training Dataset

387 A subpart of the dataset that is used to train the models to learn the relationship among 388 dependent and independent variables is called the training dataset. We divided our data into 389 training and testing using the train_test_ split function of the scikit library using python. Our 390 training dataset consists of $70 \%$ of the dataset that is more than 70,000 labeled sentences of Urdu 391 language text.

392 Testing Dataset

393 It is also the subpart of the dataset that is usually smaller than size as compared to the training 394 dataset. In our research case, we decided to use $30 \%$ of the dataset for testing and validating the 395 396

397

398

399

400

401

402 Precision $=\frac{\text { TP }}{\text { TP }+ \text { FP }}$

$403 \quad$ Recall $=\frac{\mathrm{TP}}{\mathrm{TP}+\mathrm{FN}}$ performance of classifiers. It comprises more than 30,000 instances/sentences of Urdu langue text.

$404 \mathrm{~F} 1=2 * \frac{\text { Precision } * \text { Recall }}{\text { Precision }+ \text { Recall }}$

405 Accuracy $=\frac{\mathrm{TP}+\mathrm{TN}}{\mathrm{TP}+\mathrm{TN}+\mathrm{FP}+\mathrm{FN}}$

\section{Results}

407 To evaluate our dataset, the Python package scikit-learn is used to perform event classification at 408 the sentence level. We extracted the last-4-words of each sentence and calculated the length of 409 each sentence. To obtain the best classification results we evaluated six machine learning 410 classifiers among others i.e., Decision Tree (DT), Random Forest (RF), Logistic Regression 411 (LR), Support Vector Machine (SVM), $k$-Nearest Neighbor, and Naïve Bayes Multinominal 412 (NBM).

413 We proposed three features i.e., Length, Last-4-words, and Length and Last-4-words to classify 414 sentences into different types of events (see tab. 2). The results were obtained using 'length ' as 415 the feature is shown in Tab. 4. The classifiers i.e., DT, RF, NBM, and LR showed 32\% 416 accuracies that is very low. The comparatively second feature that is Last-4-words showed better 417 results for these above-mentioned classifiers. Random Forest showed 52\% accuracy that is a 418 considerable result as an initiative for multiclass event classification in the Urdu language text. 419 The detail of results regarding other classifiers can be seen in Tab 5. 
420 We also evaluated these classifiers using another feature that is the combination of both Length

421 and Last-4-grams. It also improved the overall 1\% accuracy of the proposed system. The

422 Random Forest showed 53.00\% accuracy. The further details of accuracies of other used

423 machine learning models can be seen in Tab.6

424 The results obtained by using the above features are very low, we deiced to use the title of the

425 post as a feature to improve the performance of the system. We integrated the "Title" of the post

426 with each sentence of the same paragraph that dramatically improves the accuracy of the system.

427 We combined the "Title" of the post with other features i.e., length, and Last-4-words. The detail

428 of the highest accuracies that is obtained by the combination of these features i.e., Last-4-words,

429 length, and title are given in Tab. 7 and Tab. 8. Random forest and $k$-NN showed the highest

430 accuracies. The detail of the confusion matrix related to the proposed system (TP, FP, TN, FN) is

431 also given in Tab. 9 and Tab. 10.

432 The standard performance measuring parameters i.e., precision, recall, and f1-measure of

433 Random Forest and $k$-NN classifiers using "Title and Last-4words" as features are given in Tab.

43411 and Tab. 12 respectively. Similarly other combinations of features i.e., "Title and Length" are

435 used to enhance the accuracy of the system. The Decision Tree and Random Forest showed the

436 highest results as compared to other classifiers for this specific combination of features. A

437 detailed summary of the results related to Decision Tree and Random Forest is given in Tab. 13

438 and Tab. 14 respectively.

439 We finally presented the comparison of four classifiers that showed the highest results in fig. 3.

440 The semantics of the script written in the Urdu language is quite different from that of English

441 and Arabic Language which causes the low performance of SVM and k-NN as compared to

442 Random Forest.

443 Discussion

444 Event extraction and classification are tightly coupled with processing resources i.e., Part of

445 speech tagger ( PoS ), Text annotators, and contextual insights. Meanwhile, the usage of local

446 languages being highly preferred over social media is creating problems to analyze by existing

447 tools. Urdu is one of those languages that have a considerable number of users and a huge bulk

448 of data on social networks. It contains worthy insights that are necessary to process for different

449 purposes like to improve security, to understand the intentions, trends, and mindset of people.

450 We performed event classification written in Urdu langue text on different social media like

451 platforms. The evaluation of results is presented after analyzing multiple features i.e., Length,

452 Last-4-words, Title, and combination of all these features converged our findings to conclude

453 that length and last-4-words are basic features to classify multiclass events but showed 53\%

454 accuracy. To improve the accuracy of the proposed system, we integrated "Title" as the feature

455 with other two features i.e., Length and Last-4-words. The combination of "Title" with "Length

456 and Last-4-words" improved the performance of the proposed system and showed the highest

457 results as reported in the abstract.

458 As described in the dataset section that the dataset is imbalanced and contains multiple classes.

459 To validate the accuracy of results not only TP, FP, TN, FP reported but also the standard 
460

461

462

463

464

465

466

\section{7}

468

469

470

471

472

473

474

475

476

477

478

479

480

481

482

483

484

485

486

487

488

489

490

491

492

493

494

495

496

497

498

499

performance evaluations parameters i.e., precision, recall, and fl-measure are reported in Tab. 11, Tab. 12, Tab. 13, and Tab.14.

Furthermore, extracting and classification of events from resource-poor language is an interesting and challenging task. There are no standard (benchmark) datasets and word embedding models like Word2Vec or Glove (Exists for the English Language) for Urdu language text.

\section{Conclusions}

A massive amount of Urdu textual data exists on social networks and news websites. Multiclass event classification for Urdu text at the sentence level is a challenging task because of the few numbers of words and limited contextual information.

The selection of appropriate features and approaches is necessary to classify multiclass events written in Urdu language text.

The deep analysis of the structure of sentences written in the Urdu language leads us to select these appropriate features i.e. title, length, last-4-words, and combination of all these features.

Experimental results showed that non-of single feature is capable to classify multiclass events.

Contrary to the different combinations of these features i..e title and 4-words, title and length and last-4-words and length showed considerable results.

Count_Vectorizer and TF-IDF feature generating techniques are used to convert text into (numeric) real value for machine learning models. Random Forest classification model showed $52 \%$ and $53 \%$ accuracy for Last-4-words and combination of length and last-4-words.

The title is the key feature that can dramatically improve the performance of event classification models that works on a sentence level. Combining title with last-4-words and length showed the highest accuracies i.e. $98.00 \%$ and $99.00 \%$ for Random Forest and $k$-NN classifiers respectively.

\section{Future Work}

- In a comprehensive review of Urdu literature, we found a few numbers of referential works related to Urdu text processing. One of the main issues associated with the Urdu language research is the unavailability of the appropriate corpus like the data set of Urdu sentences representing the event; the close-domain PoS tagger; the lexicons, and the annotator, etc.

- There is a need to develop the supporting tools i.e., the PoS tagger, the annotation tools, the dataset of the Urdu-based languages having information about some information associated with the events, and the lexicons can be created to extend the research areas in the Urdu language.

- In the future, many other types of events and other domains of information like medical events, social, local, and religious events can be classified using the extension of machine learning i.e., deep learning. 
500

501

502

503

504

505

506

507

508

509

510

511

512

513

514

515

516

517

518

519

520

521

522

523

524

525

526

527

528

529

530

531

532

533

534

535

536

537

538

539

- In the future grammatical, contextual, and lexical information can be used to categorize events. Temporal information related to events can be further utilized to classify an event as real and retrospective.

- Classification of events can be performed at the document level and phrase level.

- Deep learning classifiers can be used for a higher number of event classes.

\section{Acknowledgements}

I am very grateful to the Chairman of the Faculty of Computing and Head of Department of

Software Engineering of Islamia University Bahawalpur, Pakistan. They encouraged and guided me to make research work more interesting.

\section{References}

[1] Lenhart, A., Ling, R., Campbell, S., \& Purcell, K. (2010). Teens and mobile phones: Text messaging explodes as teens embrace it as the centerpiece of their communication strategies with friends. Pew Internet \& American Life Project.

[2] Motoyama, M., Meeder, B., Levchenko, K., Voelker, G. M., \& Savage, S. (2010). Measuring Online Service Availability Using Twitter. WOSN, 10, 13-13.

[3] Reuter, T., \& Cimiano, P. (2012, June). Event-based classification of social media streams. In Proceedings of the 2nd ACM International Conference on Multimedia Retrieval (pp. 1-8).

[4] Rogstadius, J., Vukovic, M., Teixeira, C. A., Kostakos, V., Karapanos, E., \& Laredo, J. A. (2013). CrisisTracker: Crowdsourced social media curation for disaster awareness. IBM Journal of Research and Development, 57(5), 4-1.

[5] Yin, J., Lampert, A., Cameron, M., Robinson, B., \& Power, R. (2012). Using social media to enhance emergency situation awareness. IEEE Annals of the History of Computing, 27(06), 52-59.

[6] Purohit, H., Dong, G., Shalin, V., Thirunarayan, K., \& Sheth, A. (2015, December). Intent classification of short-text on social media. In 2015 ieee international conference on smart city/socialcom/sustaincom (smartcity) (pp. 222-228). IEEE.

[7] Alkhatibl, M., El Barachi, M., \& Shaalan, K. (2018, June). Using Arabic Social Media Feeds for Incident and Emergency Management in Smart Cities. In 2018 3rd International Conference on Smart and Sustainable Technologies (SpliTech) (pp. 1-6). IEEE.

[8] Khan, W., Daud, A., Nasir, J. A., \& Amjad, T. (2016). Named entity dataset for urdu named entity recognition task. Organization, 48, 282.

[9] Jacobs, I. S. (1963). Fine particles, thin films and exchange anisotropy. Magnetism, 271350.

[10] Livingston, F. (2005). Implementation of Breiman's random forest machine learning algorithm. ECE591Q Machine Learning Journal Paper, 1-13.

[11] Ghulam, S. M., \& Soomro, T. R. (2018, March). Twitter and Urdu. In 2018 International Conference on Computing, Mathematics and Engineering Technologies (iCoMET) (pp. 1-6). IEEE. 
540 [12] Naz, M., \& Hussain, S. (2013, December). Binarization and its evaluation for Urdu

541

542

543

544

545

546

547

548

549

550

551

552

553

554

555

556

557

558

559

560

561

562

563

564

565

566

567

568

569

570

571

572

573

574

575

576

577

578

579 Nastalique document images. In INMIC (pp. 213-218). IEEE.

[13] Mukund, S., Srihari, R., \& Peterson, E. (2010). An Information-Extraction System for Urdu---A Resource-Poor Language. ACM Transactions on Asian Language Information Processing (TALIP), 9(4), 1-43.

[14] Nadeau, D., \& Sekine, S. (2007). A survey of named entity recognition and classification. Lingvisticae Investigationes, 30(1), 3-26.

[15] Riaz, K. (2008, October). Concept search in Urdu. In Proceedings of the 2nd PhD workshop on Information and Knowledge Management (pp. 33-40).

[16] Ramesh, D., \& Kumar, S. S. INTERNATIONAL JOURNAL OF ENGINEERING SCIENCES \& RESEARCH TECHNOLOGY EVENT EXTRACTION FROM NATURAL LANGUAGE TEXT.

[17] Ahmed, K., Ali, M., Khalid, S., \& Kamran, M. (2016). Framework for Urdu News Headlines Classification. Journal of Applied Computer Science \& Mathematics, (21).

[18] Kong, X., Shi, X., \& Yu, P. S. (2011, April). Multi-label collective classification. In Proceedings of the 2011 SIAM International Conference on Data Mining (pp. 618-629). Society for Industrial and Applied Mathematics.

[19] Sarker, A., \& Gonzalez, G. (2015). Portable automatic text classification for adverse drug reaction detection via multi-corpus training. Journal of biomedical informatics, 53, 196207.

[20] Pedregosa, F., Varoquaux, G., Gramfort, A., Michel, V., Thirion, B., Grisel, O., ... \& Duchesnay, E. (2011). Scikit-learn: Machine learning in Python. the Journal of machine Learning research, 12, 2825-2830.

[21] Vosoughi, S., Zhou, H., \& Roy, D. (2016). Enhanced twitter sentiment classification using contextual information. arXiv preprint arXiv:1605.05195.

[22] Baker, P., Hardie, A., McEnery, T., \& Jayaram, B. D. (2003, April). Corpus data for South Asian language processing. In Proceedings of the 10th Annual Workshop for South Asian Language Processing, EACL.

[23] Becker, D., \& Riaz, K. (2002). A study in urdu corpus construction. In COLING-02: The 3rd Workshop on Asian Language Resources and International Standardization.

[24] Malik, M. K. (2017). Urdu named entity recognition and classification system using artificial neural network. ACM Transactions on Asian and Low-Resource Language Information Processing (TALLIP), 17(1), 1-13.

[25] Alsaedi, N., \& Burnap, P. (2015, April). Arabic event detection in social media. In International Conference on Intelligent Text Processing and Computational Linguistics (pp. 384-401). Springer, Cham.

[26] Al-Radaideh, Q. A., \& Al-Abrat, M. A. (2019). An Arabic text categorization approach using term weighting and multiple reducts. Soft Computing, 23(14), 5849-5863.

[27] Daud, A., Khan, W., \& Che, D. (2017). Urdu language processing: a survey. Artificial Intelligence Review, 47(3), 279-311. 
580 [28] Kuila, A., chandra Bussa, S., \& Sarkar, S. (2018). A Neural Network based Event

581

582

583

584

585

586

587

588

589

590

591

592

593

594

595

596

597

598

599

600

601

602

603

604

605

606

607

608

609

610

611

612

613

Extraction System for Indian Languages. In FIRE (Working Notes) (pp. 291-301).

[29] Singh, J. P., Dwivedi, Y. K., Rana, N. P., Kumar, A., \& Kapoor, K. K. (2019). Event classification and location prediction from tweets during disasters. Annals of Operations Research, 283(1), 737-757.

[30] Ali, A. R., \& Ijaz, M. (2009, December). Urdu text classification. In Proceedings of the 7th international conference on frontiers of information technology (pp. 1-7).

[31] Agarwal, A., \& Rambow, O. (2010, October). Automatic detection and classification of social events. In Proceedings of the 2010 Conference on Empirical Methods in Natural Language Processing (pp. 1024-1034).

[32] Sakaki, T., Okazaki, M., \& Matsuo, Y. (2010, April). Earthquake shakes twitter users: real-time event detection by social sensors. In Proceedings of the 19th international conference on World wide web (pp. 851-860).

[33] Hussain, S. (2008). Resources for Urdu language processing. In Proceedings of the 6th workshop on Asian Language Resources.

[34] Usman, M., Shafique, Z., Ayub, S., \& Malik, K. (2016). Urdu text classification using majority voting. Int. J. Adv. Comput. Sci. Appl, 7(8), 265-273.

[35] Guo, G., Wang, H., Bell, D., Bi, Y., \& Greer, K. (2003, November). KNN model-based approach in classification. In OTM Confederated International Conferences" On the Move to Meaningful Internet Systems" (pp. 986-996). Springer, Berlin, Heidelberg.

[36] Zhang, Y. (2012, September). Support vector machine classification algorithm and its application. In International Conference on Information Computing and Applications (pp. 179-186). Springer, Berlin, Heidelberg.

[37] Zhong, Y. (2016, August). The analysis of cases based on decision tree. In 2016 7th IEEE international conference on software engineering and service science (ICSESS) (pp. 142147). IEEE.

[38] Xu, S. (2018). Bayesian Naïve Bayes classifiers to text classification. Journal of Information Science, 44(1), 48-59.

[39] Zhang, T., \& Oles, F. J. (2001). Text categorization based on regularized linear classification methods. Information retrieval, 4(1), 5-31.

[40] Rustam, F., Ashraf, I., Mehmood, A., Ullah, S., \& Choi, G. S. (2019). Tweets classification on the base of sentiments for US airline companies. Entropy, 21(11), 1078.

[41] Ali, D., Missen, M. M. S., \& Husnain, M. (2021). Multiclass Event Classification from Text. Scientific Programming, 2021. 


\section{Table $\mathbf{1}$ (on next page)}

Proposed Features 
1

2
Table 1: Proposed Features

\begin{tabular}{ll} 
Sr. No. & Feature Name \\
1 & Length \\
2 & Last_4_words \\
3 & Last_4_words and Length \\
4 & Title \\
5 & Title and Length \\
6 & Title and Last_4-words \\
\hline
\end{tabular}




\section{Table 2 (on next page)}

Types of events and their labels in the dataset 
1

2

3
Table 2: Types of events and their labels in the dataset

\begin{tabular}{llll}
\hline Event & Label & Event & Label \\
\hline Sports & 1 & Earthquake & 7 \\
Inflation & 2 & Showbiz & 8 \\
Murder and Death & 3 & Fraud and Corruption & 9 \\
Terrorist Attack & 4 & Rain/Weather & 10 \\
Politics & 5 & Sexual Assault & 11 \\
Law and Order & 6 & Business & 12 \\
\hline
\end{tabular}




\section{Table 3(on next page)}

Last 4-words representing an event 
1

2

Table 3: Last 4-words representing an event

\begin{tabular}{|c|c|c|c|}
\hline \multicolumn{2}{|c|}{ Event } & \multicolumn{2}{|c|}{ Non_Event } \\
\hline Urdu & English & Urdu & English \\
\hline 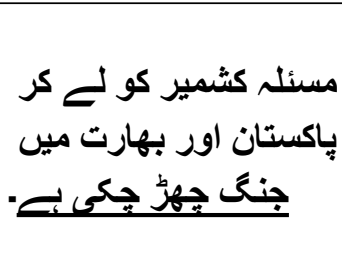 & $\begin{array}{l}\text { The battle between } \\
\text { Pakistan and India } \\
\text { has been started on } \\
\text { the conflict of } \\
\text { Kashmir. }\end{array}$ & جنا دن يهلح لوى خوش ته & $\begin{array}{l}\text { Few days ago, } \\
\text { people were happy. }\end{array}$ \\
\hline
\end{tabular}

3 


\section{Table 4 (on next page)}

Length 
1

2

3

4

5
Table 4: Length

\begin{tabular}{lll}
\hline Algorithms & Accuracy & Feature \\
\hline SVM & $17 \%$ & \\
NBM & $32 \%$ & \\
LR & $32 \%$ & Length \\
Decision Tree & $32 \%$ & \\
Random Forest & $32 \%$ & \\
K-NN & $24 \%$ & \\
\hline
\end{tabular}




\section{Table 5 (on next page)}

Last _4_words accuracy 
1

2

3
Table 5: Last_4_words accuracy

\begin{tabular}{lll}
\hline Algorithms & Accuracy & Feature \\
\hline SVM & $45 \%$ & \\
NBMN & $44 \%$ & \\
LR & $49 \%$ & Last_4_words \\
Decision Tree & $49 \%$ & \\
Random Forest & $\mathbf{5 2 \%}$ & \\
K-NN & $48 \%$ & \\
\hline
\end{tabular}




\section{Table 6 (on next page)}

Last _4_words and Length Accuracy 
1

2

3
Table 6: Last_4_words and Length Accuracy

\begin{tabular}{lll}
\hline Algorithms & Accuracy & Feature \\
\hline SVM & $46 \%$ & \\
NBMN & $44 \%$ & \\
LR & $49 \%$ & \\
Decision Tree & $48 \%$ & Length and \\
Random & $\mathbf{5 3 \%}$ & Last_4_words \\
Forest & & \\
K-NN & $49 \%$ & \\
\hline
\end{tabular}




\section{Table 7 (on next page)}

Title and Last_4_words accuracy 
1

2

3
Table 7: Title and Last_4_words accuracy

\begin{tabular}{lll}
\hline Algorithms & Accuracy & Feature \\
\hline SVM & $85 \%$ & \\
NBMN & $91 \%$ & \\
LR & $95 \%$ & Title and Last \\
Decision Tree & $97 \%$ & -4 _words \\
Random & $\mathbf{9 8 \%}$ & \\
Forest & & \\
K-NN & $\mathbf{9 9 \%}$ & \\
\hline
\end{tabular}




\section{Table 8 (on next page)}

Title and Length 
1

2

3

4
Table 8: Title and Length

\begin{tabular}{lll}
\hline Algorithms & Accuracy & Feature \\
\hline SVM & $87 \%$ & \\
NBMN & $93 \%$ & \\
LR & $98 \%$ & Title and \\
Decision Tree & $\mathbf{9 9 \%}$ & Length \\
Random & $\mathbf{9 9 \%}$ & \\
Forest & & \\
K-NN & $94 \%$ & \\
\hline
\end{tabular}




\section{Table 9 (on next page)}

Random forest TP, FN, FP and TN 


\begin{tabular}{|c|c|c|c|c|c|}
\hline \multirow[b]{2}{*}{ Label } & & & & & \\
\hline & Type of Event & $\mathrm{TP}$ & $\mathrm{FN}$ & FP & $\mathrm{TN}$ \\
\hline 1 & Sports & 5646 & 15 & 14 & 25514 \\
\hline 2 & Inflation & 967 & 0.0 & 08 & 30211 \\
\hline 3 & $\begin{array}{l}\text { Murder and } \\
\text { Death }\end{array}$ & 2096 & 19 & 22 & 29052 \\
\hline 4 & Terrorist Attack & 865 & 13 & 06 & 30304 \\
\hline 5 & Politics & 9983 & 47 & 86 & 21073 \\
\hline 6 & law and order & 2257 & 36 & 23 & 28872 \\
\hline 7 & Earthquake & 970 & 0.0 & 0.0 & 30219 \\
\hline 8 & Showbiz & 2244 & 15 & 04 & 28929 \\
\hline 9 & $\begin{array}{l}\text { Fraud and } \\
\text { corruption }\end{array}$ & 3015 & 29 & 21 & 35924 \\
\hline 10 & Rain/weather & 1031 & 0.0 & 05 & 34888 \\
\hline 11 & Sexual Assault & 889 & 0.0 & 01 & 30300 \\
\hline 12 & Business & 1032 & 20 & 04 & 30134 \\
\hline
\end{tabular}


Table $\mathbf{1 0}$ (on next page)

KNN TP, FN, FP and TN 


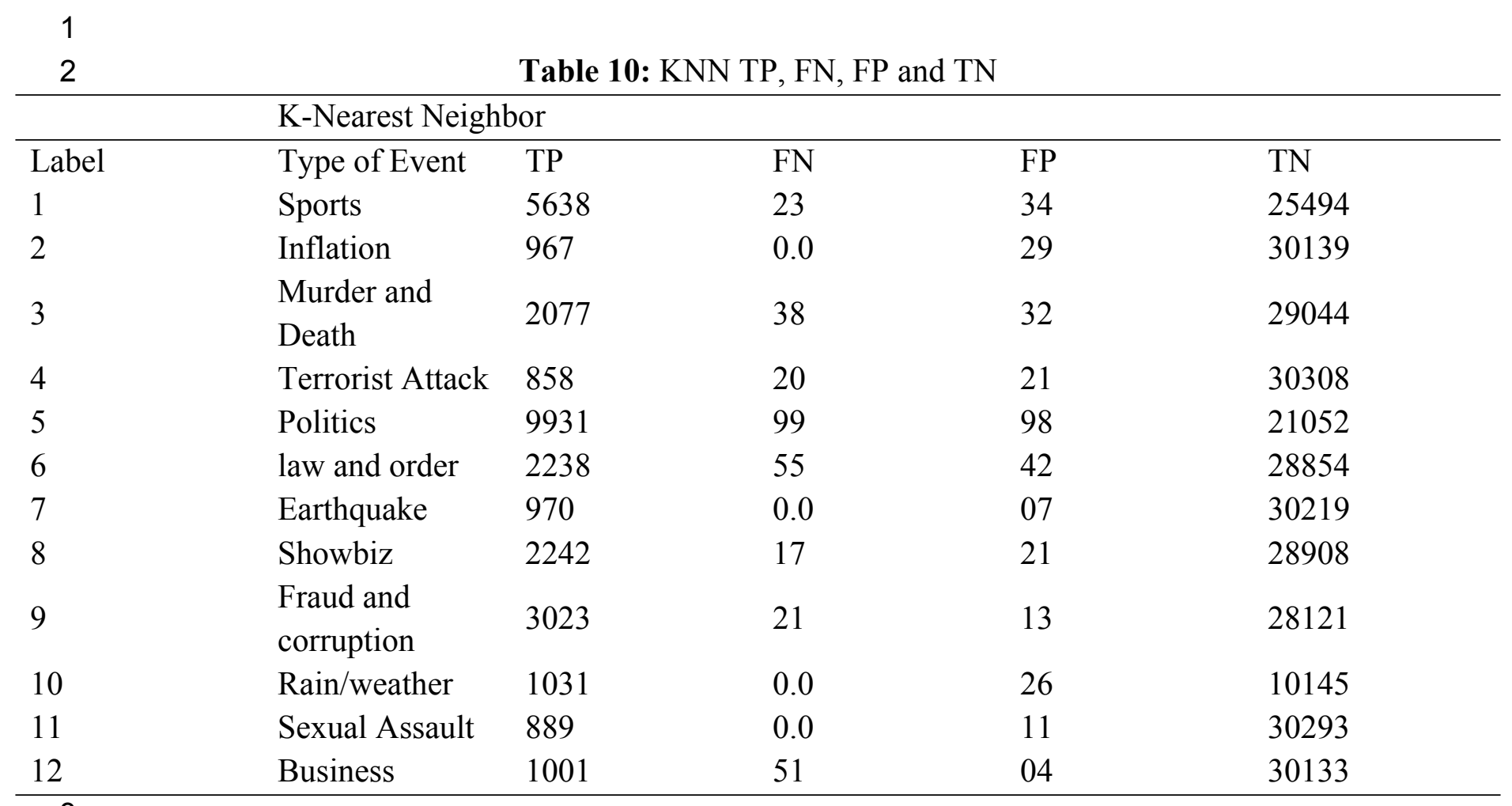




\section{Table 11 (on next page)}

Random Forest performance using the title, and last_4_words 
1

2

Table 11: Random Forest performance using the title, and last_4_words

\begin{tabular}{lllll}
\hline Label & Event & Precision & Recall & F1_Measure \\
\hline 1 & Sports & 0.99 & 0.99 & 0.99 \\
2 & Inflation & 0.99 & 1.00 & 0.99 \\
3 & Murder and Death & 0.98 & 0.99 & 0.98 \\
4 & Terrorist Attack & 0.97 & 0.96 & 0.97 \\
5 & Politics & 0.98 & 0.99 & 0.98 \\
6 & law and order & 0.98 & 0.96 & 0.97 \\
7 & Earthquake & 1.00 & 1.00 & 1.00 \\
8 & Showbiz & 0.99 & 0.98 & 0.99 \\
9 & Fraud and corruption & 0.99 & 0.98 & 0.98 \\
10 & Rain/weather & 1.00 & 1.00 & 1.00 \\
11 & Sexual Assault/Intercourse & 1.00 & 1.00 & 1.00 \\
12 & Business & 0.98 & 0.95 & 0.97 \\
Overall accuracy & $98.53 \%$ & & & \\
\hline
\end{tabular}

3 


\section{Table $\mathbf{1 2}$ (on next page)}

KNN performance using the title, and last_4_words 
1

Table 12: KNN performance using the title, and last_4_words

\begin{tabular}{lllll}
\hline Label & Event & Precision & Recall & F1_Measure \\
\hline 1 & Sports & 0.99 & 1.00 & 0.99 \\
2 & Inflation & 0.97 & 1.00 & 0.99 \\
3 & Murder and Death & 0.99 & 0.98 & 0.98 \\
4 & Terrorist Attack & 0.98 & 0.98 & 0.98 \\
5 & Politics & 0.99 & 0.99 & 0.99 \\
6 & law and order & 0.98 & 0.98 & 0.98 \\
7 & Earthquake & 1.00 & 1.00 & 1.00 \\
8 & Showbiz & 0.99 & 0.99 & 0.99 \\
9 & Fraud and corruption & 0.99 & 0.99 & 0.99 \\
10 & Rain/weather & 0.99 & 1.00 & 0.99 \\
11 & Sexual Assault/Intercourse & 0.99 & 1.00 & 1.00 \\
12 & Business & 1.00 & 0.95 & 0.97 \\
Overall accuracy & $98.96 \%$ & & & \\
\hline
\end{tabular}

2 


\section{Table $\mathbf{1 3}$ (on next page)}

Decision Tree performance using the 'Title and Length' 
1

Table 13: Decision Tree performance using the 'Title and Length'

\begin{tabular}{lllll}
\hline Label & Event & Precision & Recall & F1_Measure \\
\hline 1 & Sports & 1.00 & 1.00 & 1.00 \\
2 & Inflation & 1.00 & 1.00 & 1.00 \\
3 & Murder and Death & 0.99 & 0.99 & 0.99 \\
4 & Terrorist Attack & 0.99 & 0.99 & 0.99 \\
5 & Politics & 1.00 & 1.00 & 1.00 \\
6 & law and order & 0.99 & 1.00 & 0.99 \\
7 & Earthquake & 1.00 & 1.00 & 1.00 \\
8 & Showbiz & 1.00 & 0.99 & 1.00 \\
9 & Fraud and corruption & 1.00 & 0.99 & 1.00 \\
10 & Rain/weather & 1.00 & 1.00 & 1.00 \\
11 & Sexual Assault/Intercourse & 1.00 & 1.00 & 1.00 \\
12 & Business & 1.00 & 0.98 & 0.99 \\
Overall accuracy & $99.63 \%$ & & & \\
\hline
\end{tabular}

2 


\section{Table 14(on next page)}

Random Forest performance using the 'Title and Length' 
1

Table 14: Random Forest performance using the 'Title and Length'

\begin{tabular}{lllll}
\hline Label & Event & Precision & Recall & F1_Measure \\
\hline 1 & Sports & 1.00 & 1.00 & 1.00 \\
2 & Inflation & 1.00 & 1.00 & 1.00 \\
3 & Murder and Death & 1.00 & 1.00 & 1.00 \\
4 & Terrorist Attack & 1.00 & 0.99 & 1.00 \\
5 & Politics & 1.00 & 1.00 & 1.00 \\
6 & law and order & 1.00 & 1.00 & 1.00 \\
7 & Earthquake & 1.00 & 1.00 & 1.00 \\
8 & Showbiz & 1.00 & 1.00 & 1.00 \\
9 & Fraud and corruption & 1.00 & 1.00 & 1.00 \\
10 & Rain/weather & 1.00 & 1.00 & 1.00 \\
11 & Sexual Assault/Intercourse & 1.00 & 1.00 & 1.00 \\
12 & Business & 1.00 & 1.00 & 1.00 \\
Overall accuracy & $99.92 \%$ & & & \\
\hline
\end{tabular}

2 
Figure 1

Concept Diagram

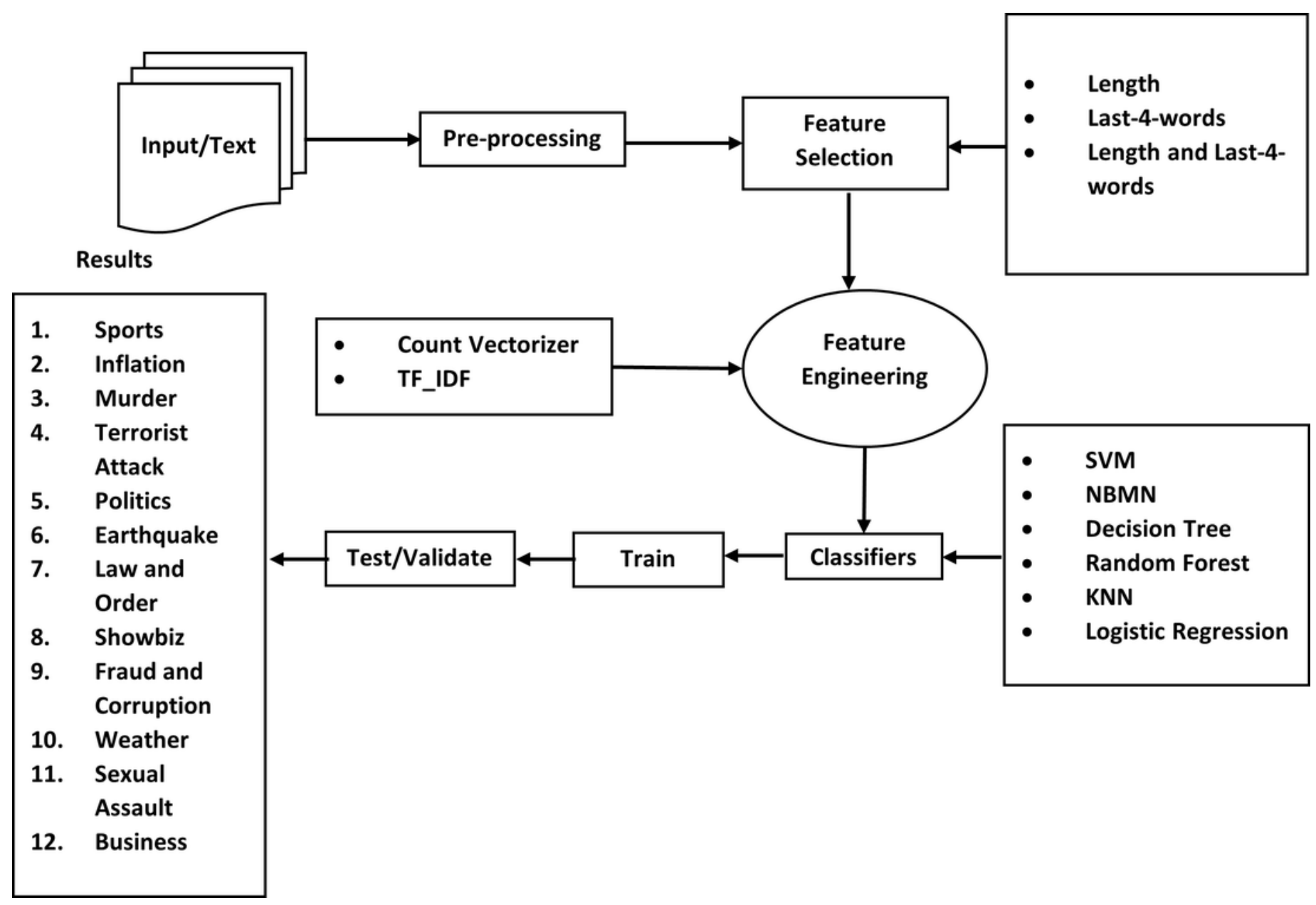


Figure 2

Maximum Number of Instances

\section{Imblance instance based Multi-Class Dataset}

\section{0}

35000

30000

25000

20000

15000

10000

5000

0
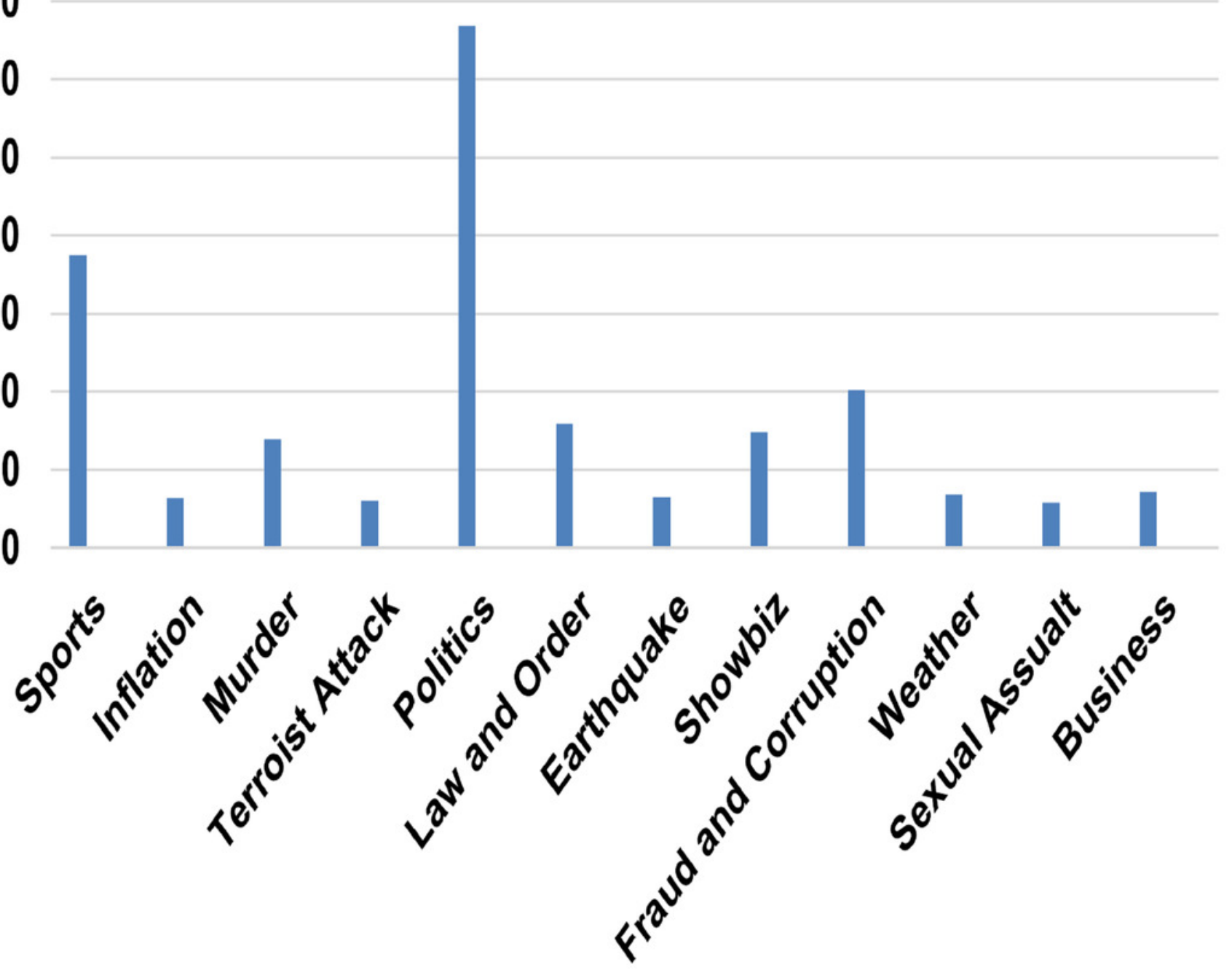

Figure 2: Maximum number of instances 
Figure 3

The Best features and the best classi $\square$ ers

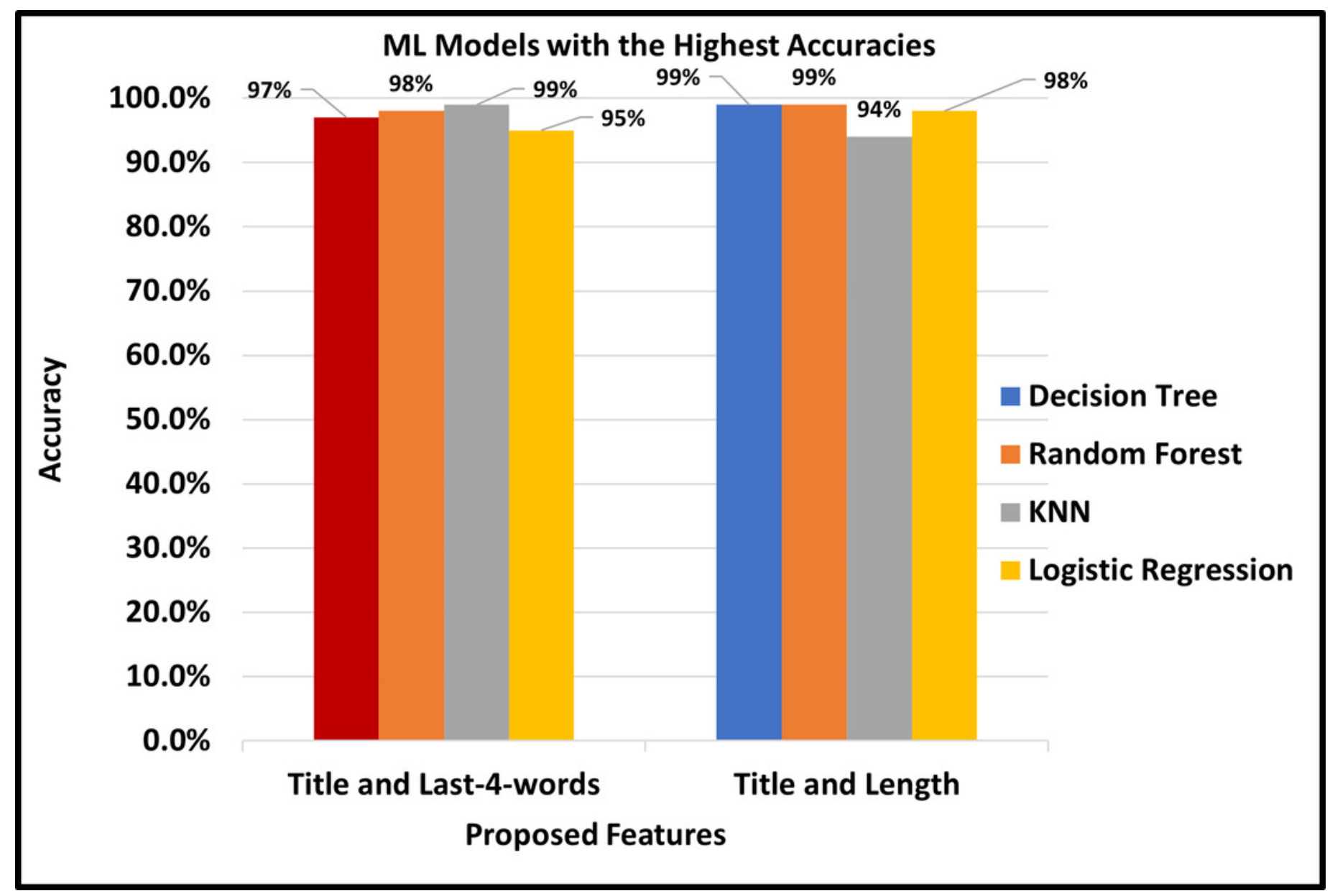

